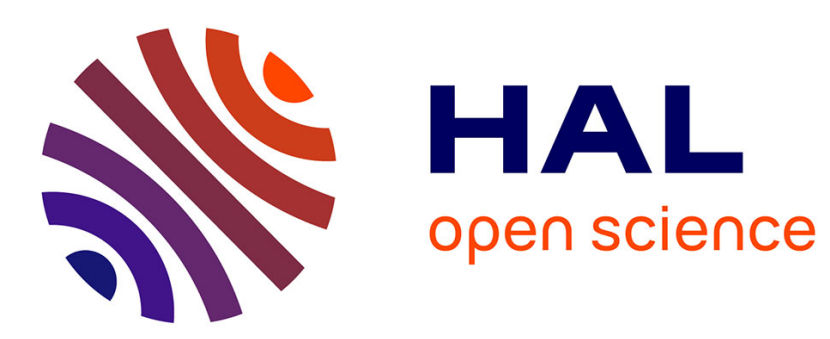

\title{
Les visualisations de données inspirées par le jeu et la conception par disengamement \\ Stéphane Goria
}

\section{To cite this version:}

Stéphane Goria. Les visualisations de données inspirées par le jeu et la conception par disengamement. Les Cahiers du numérique, 2016, La visualisation de données, 12 (4), pp.39-64. 10.3166/lcn.12.4.3964. hal-02266835

\section{HAL Id: hal-02266835 \\ https://hal.science/hal-02266835}

Submitted on 12 Dec 2019

HAL is a multi-disciplinary open access archive for the deposit and dissemination of scientific research documents, whether they are published or not. The documents may come from teaching and research institutions in France or abroad, or from public or private research centers.
L'archive ouverte pluridisciplinaire HAL, est destinée au dépôt et à la diffusion de documents scientifiques de niveau recherche, publiés ou non, émanant des établissements d'enseignement et de recherche français ou étrangers, des laboratoires publics ou privés. 


\section{Les visualisations de données inspirées par le jeu et la conception par disengamement}

STEPHANE GORIA

\section{Introduction}

Il existe désormais de nombreuses catégories d'applications correspondant à des cas d'inspirations, d'emplois ou de détournements du jeu pour atteindre des fins non ludiques. Certaines sont maintenant très connues comme la conception et l'usage de serious games. Nous souhaitons aborder dans cet article un cas moins connu d'emploi du jeu à des fins sérieuses, mais dont le potentiel nous semble très important : le disengamement. Nous considérons que, du point de vue de la visualisation de données, le disengamement donne des possibilités très intéressantes et de nouvelles perspectives en termes de conception de supports de présentation notamment cartographiques.

Mais avant d'aborder en détail cette notion, il nous semble qu'un parcours des grandes catégories d'applications sérieuses que l'on peut actuellement lier au jeu s'impose. Cela nous permettra de mieux positionner le disengamement par rapport à ces autres applications sérieuses inspirées par le jeu. Ceci fait, à partir d'un exemple, nous aborderons la mise en œuvre des principes de disengamement pour montrer comment ils peuvent être appliqués dans une perspective de cartographie d'information. Il s'agira de répondre aux questions suivantes: comment peut-on réaliser une cartographie à partir d'un jeu et quel peut être son apport ? Enfin, sur la base de quelques retours d'expériences auprès de public constitués d'étudiants, de chercheurs et de professionnels nous conclurons cette présentation.

\section{Différentes formes d'utilisation du jeu à des fins sérieuses}

Depuis quelques années les jeux ne sont plus perçus et abordés seulement pour leurs caractéristiques ludiques et distractives, mais aussi en termes d'atouts et de potentiels pour intéresser certains usagers ou personnes, au moins, à des fins de formation ou d'information. Ainsi, depuis le début des années 2000 différents vocabulaires ont fait leur apparition et se sont répandus dans le milieu de l'entreprise. Parmi ces derniers, certains sont plus présents que d'autres tels que : serious game, game with a purpose, serious gaming et gamification. Toutes ces formes sont encore en émergences et en cours de distinction les unes par rapport aux autres. Aussi, avant d'aborder une autre forme complémentaire de conception et d'utilisation d'outils inspirés par le jeu, il nous semble important de donner quelques éléments de compréhension des catégories déjà établies. Nous les présenterons donc les unes en fonction des autres, tout en les liant à des questions de visualisation de données. 


\subsection{Les serious games}

L'expression « serious game » ou « jeu sérieux » ou encore « jeu utilitaire » est apparue au tout début des années 1970 et a d'abord été inspirée par le potentiel des jeux de plateau pour la formation (Abt, 1970). Elle fut employée de manière plus ou moins anecdotique durant une vingtaine d'années dans le domaine militaire qui lui préfère, pendant quelque temps, l'expression «wargame» (Perla, 1990, 2) et celui de la formation des enfants de «jeu ludoéducatif » (Berry, 2011), même si ces deux expressions ne recouvrent qu'une partie des emplois possibles des serious games. Avec le développement d'une nouvelle génération de jeux vidéo sur consoles et de jeu de simulation sur PC durant les années 1990s, le nombre de serious games conçus ou dérivés pour la formation militaire augmente et le succès rencontré sont mis en valeur à la fin de cette décennie (Smith, 2009, 213). Ceci s'explique aussi par les transferts de technologies qui ont lieu lors de cette décennie entre les industriels travaillant pour l'armée américaine et quelques grands studios de jeu vidéo comme SEGA et NAMCO (Berget, 2015). Après quelques succès surtout dans le domaine militaire (Smith, 2009, 2012), le serious game prend véritablement son essor au tout début des années 2000s et se répand dans toutes les sphères de l'entreprise et de reste de la société. Dès lors, cette catégorie de dispositifs inspirés par les jeux correspond ainsi à tout outil assimilable à un jeu et conçu en tant que tel dans l'objectif de permettre la réalisation d'une tâche non ludique et qualifiée de sérieuse. Étant donné la grande contribution des jeux vidéo au développement des serious games, certains auteurs ajoutent à cette définition le fait qu'un serious game doit comporter une interface numérique (Alvarez et Djaouti, 2012, 12).

Toutefois, si nous parlons d'outil assimilable à un jeu, ce n'est pas le qualificatif employé par les concepteurs de serious games qui parlent simplement de jeux développés pour parvenir à une fin sérieuse. Les chercheurs et autres spécialistes du jeu qui, en majorité, font partie d'un courant de recherche souvent nommé "game studies" ne considèrent d'ailleurs pas véritablement les serious games comme des jeux et une partie de leurs travaux interroge encore sur la pertinence de cette appellation de serious game (Brougère, 2010). En effet, pour les puristes du jeu qui se fondent sur les réflexions de R. Caillois (1967) et J. Huizinga (1951), un véritable jeu se doit d'être improductif et laisser le joueur libre d'intégrer le jeu comme d'en sortir ou de ne pas y jouer. Ce ne peut pas être le cas des serious games. Dès leur conception, ils sont pensés à des fins de production. Seuls les jeux dédiés à l'apprentissage pourraient s'interpréter comme des artefacts de transferts de connaissances et non comme des outils de production de connaissances. Dans ce cas, ils peuvent être nommés jeux. En fait, tous les serious games sont conçus et déployés pour réaliser des tâches propres à une organisation que l'on peut généralement qualifier de « travail ». Or, s'il s'agit d'une tâche relevant du travail, celle-ci ne peut être que trop rarement considérée comme libre au sens d'un jeu. Les jeux ludo-éducatifs ne font pas exception à cette règle puisqu'ils permettent un travail d'apprentissage dans un contexte scolaire. Les serious games sont donc des jeux, si on les analyse à partir de leurs caractéristiques physiques de support de jeu et leurs traits de ressemblance avec des choses qui sont bel et bien des jeux. Par contre, ils s'en distinguent par les contraintes contextuelles de pratiques du jeu.

Actuellement, les serious games peuvent être classés selon les tâches qu'ils permettent d'accomplir. On peut ainsi répertorier plusieurs sous-catégories de serious games selon l'objectif visé qui peut être: l'entrainement (exergames), la formation (edugames), la publicité (advergames), l'acquisition de données (datagames), l'information de type journalistique (newsgames), etc. (Alvarez et Djaouti, 2012, 21). Par exemple, le newsgame September 12th, est 
Les visualisations de données inspirées par le jeu 3

un jeu sérieux élaboré par G. Frasca (2006) qui a été conçu afin de passer un message d'actualité puisque sa conception date du début des bombardements en Afghanistan suite à la tragédie du 11 septembre 2001. Afin de faire comprendre au joueur que les bombardements ne peuvent être solution contre le terrorisme, le jeu est présenté sous la forme d'une vue du ciel d'une ville du Moyen-Orient, dans laquelle se promènent des personnes dont quelques terroristes sur lesquels on peut tirer puisque le curseur du joueur est une cible. Si le joueur clique, un missile est envoyé à l'endroit visé, mais si des terroristes sont tués par le missile (figure 1), inévitablement des innocents le sont aussi, ce qui a pour conséquence la création de nouveaux terroristes.

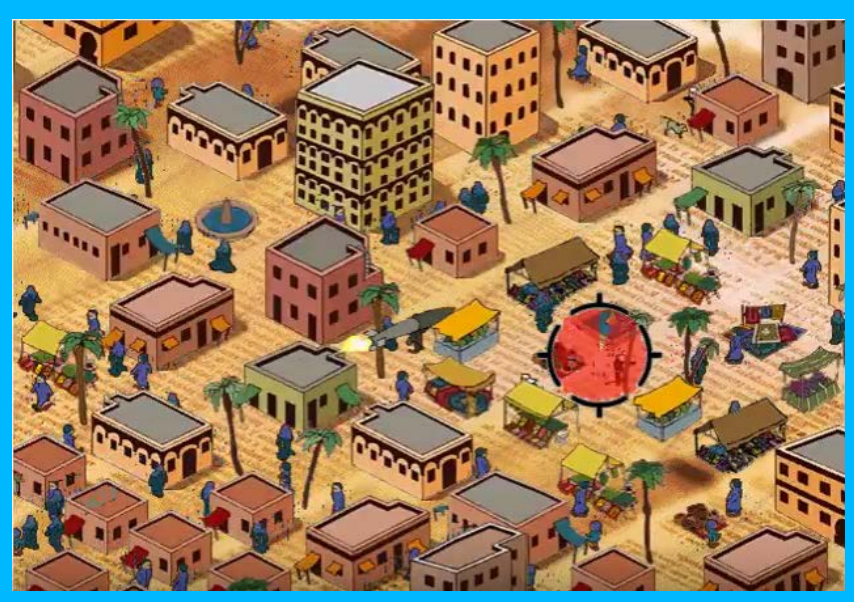

Figure 1. September $12^{\text {th }}$

Abordé, dans la perspective d'une visualisation de données qui nous intéresse tout particulièrement ici, un serious game est une solution qui répond à un problème posé de la manière suivante: "quel jeu peut-on développer afin de répondre à un problème de visualisation de données?».

Il doit être abordé en ce sens et son développement comme celui de tout jeu doit impliquer de répondre à des questionnements relevant du design de jeu. Dans ce cadre, il doit être conçu à la fois en termes d'histoire, de supports technologiques, d'éléments esthétiques et mécaniques de jeu. Le tout dépendant du public cible et de l’objectif sérieux visé.

\subsection{Les games with a purpose}

Cette appellation permet de qualifier une sous-catégorie de serious games quelquefois appelée datagames (voir ci-dessus) dont l'importance ne cesse de croître. Ces domaines d'application sont tout aussi varié que ceux de l'ensemble des serious games: médecine, biologie, informatique, linguistique, documentation, etc. Après quelques années de développement et d'emploi, il semble que l'appellation game with a purpose soit préférée par la communauté scientifique à celle de datagame. Par exemple, début 2016, posées sur Google Scholar les requêtes " "datagame" » et " "datagames" » ont donné respectivement 21 et 10 réponses, tandis que les requêtes " "game with a pupose" » et " "games with a pupose" » ont permis d'obtenir respectivement 684 et 2500 réponses.

Ces jeux sont parfois abrégés « gwap » et sont aussi appelés « jeux avec but » en français. A la différence des autres serious games, les gwap sont conçus pour être directement associés à un 
processus de collecte et de traitement de données. Il peut s'agir d'une aide à la production, à l'identification, à la collecte, à l'annotation, au tri, à l'indexation, à l'analyse, à l'exploration de possibilités / parcours/ solutions, etc. Alors que les autres serious games sont souvent des outils de transferts de connaissances du jeu vers le joueur, les gwap sont utilisés comme moyens de transfert de connaissances des joueurs vers une personne tiers via le jeu. De plus, la différence des autres serious games de nombreux gwap sont conçus afin de résoudre des problèmes dont les concepteurs n'ont pas la réponse, mais la recherchent (Lafourcade et al., 2015, 15). Dès lors, afin d'augmenter leur capacité d'acquisition de connaissances et de motivation de personnes pouvant les aider à résoudre leur problème, les gwap sont, très souvent, fondés sur le principe du crowdsourcing : ils font appel à un très grand nombre de personnes pour réaliser une tâche utilisant pour cela des ressorts et outils du Web 2.0 tels que la constitution ou sollicitation de réseaux sociaux.

Le principe fonctionnement des gwap a été imaginé par Luis Von Ahn. Il a d'abord été le créateur des logiciels dits captchas dont le rôle consiste à vérifier si une interface informatique est utilisée par être humain ou un programme. Cela permet de limiter, par exemple, les spams ou le pillage de données par des programmes informatiques purement automatiques. Les captchas prennent la forme de suites de caractères écrites manuellement qu'un être humain est censé reconnaître facilement : l'être humain sollicité par un captcha doit recopier dans un champ dédié la suite de caractères qui lui est présentée sous forme d'image. Après avoir conçu les captchas Luis Von Ahn développa les recaptchas qui sont des captchas fondés sur le principe suivant : les séries de caractères proposées viennent de véritables écrits non numériques et sont réparties en deux parties ; l'une dont le logiciel connait le résultat et qui sert de captcha pour l'utilisateur et l'autre dont le logiciel ne connait pas le résultat et avec laquelle. En reconnaissant la partie inconnue du logiciel de la série de caractères présentés, l'utilisateur contribue en identifiant les caractères à améliorer, par exemple, les performances de logiciels de reconnaissance automatique de caractères. Plus une séquence inconnue est reconnue de manière identique par de nombreux utilisateurs différents et plus on peut être certains de la fiabilité des données ainsi collectées. Le temps perdu par les utilisateurs pour répondre à des captchas est donc réinvesti pour d'autres développements (Lafourcade et al, 2015,10). Suite aux bons résultats obtenus par les recaptchas, Luis von Ahn aborda la collecte de données par les utilisateurs en faisant appel cette fois au jeu : l'utilisateur tout en jouant contribue à un processus de collecte et de traitement de données (Von Ahn \& Dabbish, 2008).

D'autres informaticiens ont suivi la voie ouverte par Luis Von Ahn et ont rencontré de très grands succès comme avec le gwap Foldit. Ce gwap est en effet emblématique de toute la catégorie. Présenté sous la forme d'un jeu puzzle game à la Candy Crush, les joueurs sont confrontés à des problèmes de pliages de protéines à réaliser en 3 dimensions. Ces problèmes sont classés par difficulté croissante, mais relèvent de cas biomédicaux concrets. Une structure gagnante est celle qui se conforme à l'énergie nécessaire à la forme prise par l'assemblage de protéines ainsi qu'au fait qu'elle ne donne aucune aux virus (figure 2). Jusqu'à un certain niveau, tous les problèmes proposés disposent d'une solution connue, mais le dernier problème posé n'a pas de solution identifiée. Une fois Foldit mis à disposition des joueurs sur le Web, en l'espace de quelques semaines, un joueur a trouvé intuitivement une solution au dernier problème pliage sur lequel bloquaient depuis des années des équipes de chercheurs et des outils de simulations du monde entier (Cooper et al, 2010). Foldit est toujours disponible en ligne. Seul le dernier problème proposé a changé et est changé en fonction des découvertes des joueurs faisant ainsi avancer la recherche biomédicale. 


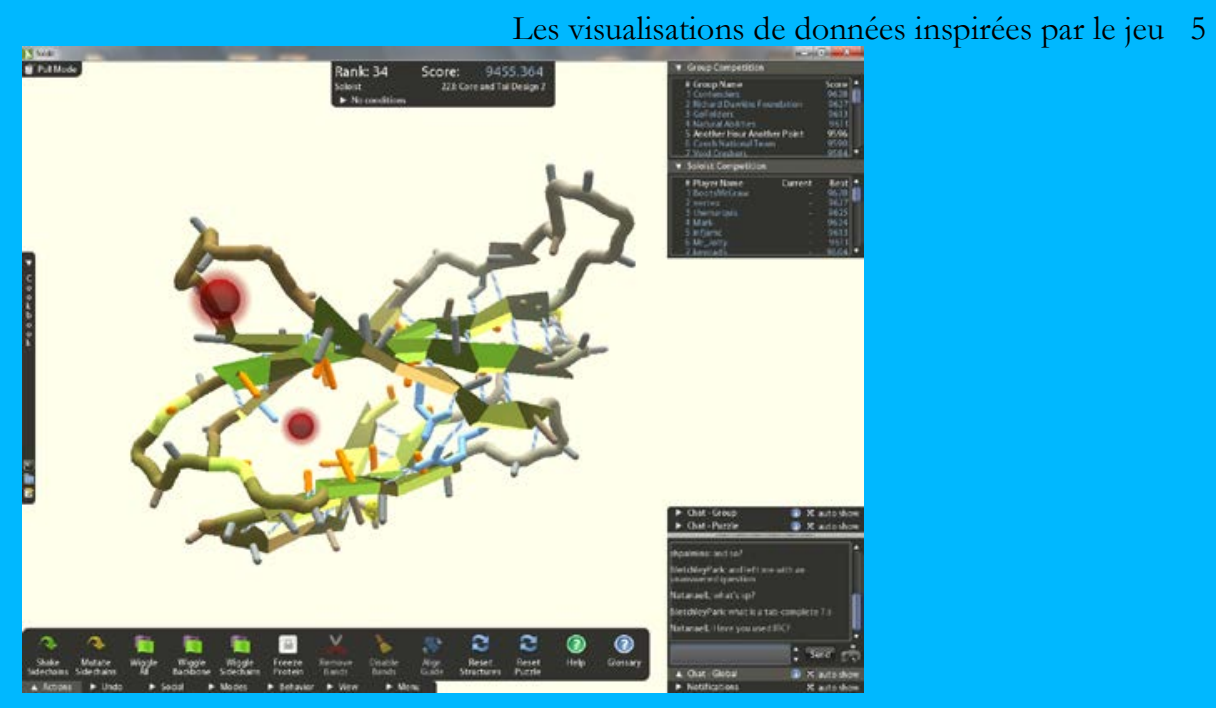

Figure 2. Légende figure sous la figure

Les gwap sont des serious games, car ils sont conçus pour être présentés comme des jeux afin d'atteindre un objectif sérieux. Cependant, on peut aussi en considérer certains comme Foldit en les assimilant à des outils professionnels transformés en jeu sérieux à l'aide d'un d'éléments de design relevant d'un processus de gamification (voir plus loin). La différence n'est pas bien importante, si le résultat final permet d'atteindre le ou les objectifs visés. Dans la perspective d'une visualisation de données, un gwap est une solution qui répond à un problème posé de la manière suivante: "quelle présentation du problème sous la forme d'un jeu permettra de collecter les données nécessaires à sa résolution?».

Ce problème doit être abordé en plusieurs étapes. Il est d'abord nécessaire de bien traduire le problème ou d'associer la solution recherchée en un problème de collecte ou de traitement de données. Ensuite, il faut imaginer un jeu qui permettra cette collecte et ce traitement en identifiant le nombre de personnes qu'il sera nécessaire d'inciter à jouer. Il faut de plus envisager la meilleure solution pour sélectionner les données les plus pertinentes parmi celles collectées. Enfin, il faut trouver des moyens pour faire connaître le jeu et intéresser les joueurs visés.

\subsection{Le serious play ou serious gaming}

Il y a deux manières d'interpréter l'expression serious gaming. En France, depuis les travaux de Alvarez et Djaouti $(2012,11)$, elle semble plutôt exprimer le fait de détourner, pour atteindre une fin sérieuse, un jeu conçu au départ à des fins purement ludiques. Pour certains auteurs anglophones, elle peut simplement exprimer la pratique de serious games (Grietzer et al, 2007 ; Crookall, 2010). Étant donné qu'en anglais, le jeu en tant que support à la mise en œuvre de l'activité ludique est nommé game et que l'activité de jeu est qualifiée de play, les anglophones préfèrent souvent employer l'expression « serious play » pour rendre compte de cas d'emplois de véritables jeux pour atteindre des objectifs sérieux. Le serious play peut prendre autant de formes qu'il y a de détournements possibles. Mais, s'il y a adaptation du jeu, celle-ci doit être assez subtile pour qu'un observateur ne voit pas la différence entre le jeu original et le jeu modifié à des fins sérieuses. De ce point de vue, le jeu est qualifié à partir de son support et du 
6 Les cahiers du numérique -

jugement qui est porté sur ce dernier par un observateur. Il s'agit d'une définition héritée d'un auteur important pour la communauté francophone qui s'intéresse au jeu : J. Henriot (1969).

Quelques fois, le support de jeu en lui-même n'est pas modifié. C'est le cas des applications du jeu de Lego à des fins de créativité et autres activités liées au management d'équipe (Frick et al, 2013). D'autres fois, un jeu est modifié par les concepteurs du jeu originel afin d'en préserver la nature et seules certaines modifications, de scénario ou de décors, par exemple, sont réalisées. C'est le cas du jeu SimCityEdu, il s'agit d'une adaptation du célèbre jeu SimiCity pour forme des enfants à la maîtrise de l'environnement (Weitze, 2014) (figure 3).

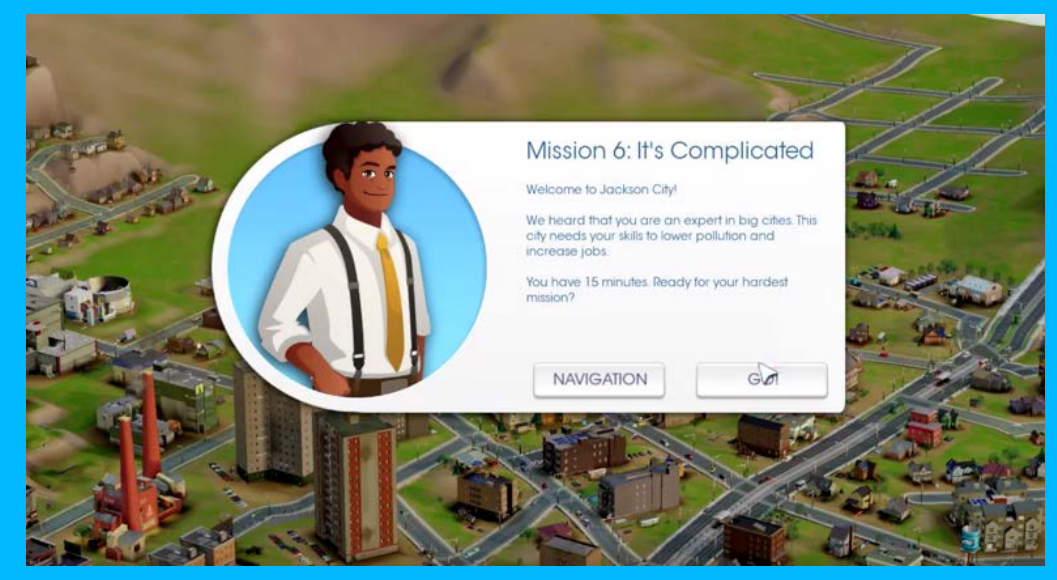

Figure 3. SimCityEdu

Dans certains cas, la distinction entre le jeu modifié et un serious game est assez subtile. C'est le cas du célèbre serious game America's Army qui en en fait inspiré du jeu vidéo Unreal Tournament dont il utilise la carte graphique à des fins de recrutement de l'armée américaine (Smith, 2009, 2013). Il est ainsi difficile, sans en connaître la nature, de juger qu'il s'agit d'un jeu vidéo du type Call of Duty ou Counter Strike ou bien d'un serious game (Figure 4), sachant qu'en plus America's Army qui peut être joué avec une PlayStation 2 ou une $X$-box.

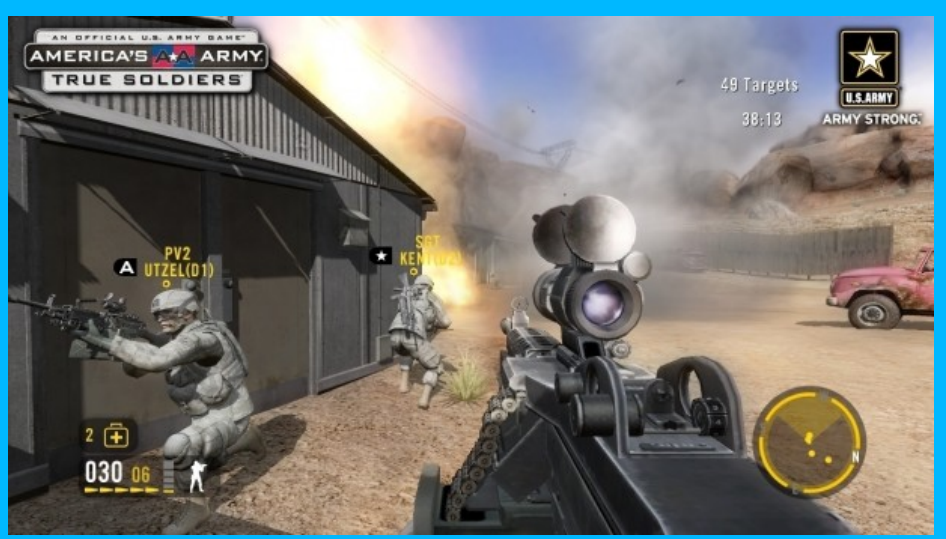

Figure 4. America's Army 
Les visualisations de données inspirées par le jeu 7

Du point de vue de la résolution de problème via une visualisation de données, un serious play est une solution qui doit être abordée de façon à répondre à un problème posé de la manière suivante: "quel jeu déjà existant peut-on détourner pour à un problème de visualisation de données?».

La réponse à cette question peut être abordée à partir de l'exposé du problème et d'un brainstorming dont le but consiste à identifier différents jeux qui pourraient convenir. La mise en œuvre d'un serious play ne doit pas nécessiter des développements importants, mais le test d'un ou plusieurs jeux. Un jeu sélectionné peut l'être, car il répond à une partie du problème et parce qu'il est plus facile à appréhender par tel public lié eu problème.

\subsection{La gamification}

La gamification qui est aussi appelée en français ludification est d'abord un courant de pensée issu des champs du Marketing et des Ressources Humaines (Silva, 2013). Mais, les résultats encourageants obtenus dans ces deux domaines ont depuis largement incité à étendre ses applications à bien d'autres domaines.

Le courant de la gamification s'est développé à partir de la seconde moitié des années 2000 et doit, sûrement, une partie de l'intérêt qu'il a suscité aux succès reportés par les serious games. Le principe de la gamification est simple : s'inspirer de la conception de jeux pour enrichir et augmenter l'intérêt ou améliorer les performances des individus pour réaliser une tâche (Muletier et al, 2014,11). Il ne s'agit pas forcément de transformer un outil ou une tâche en un jeu, mais de compléter sa mise en œuvre ou conception par des aspects ou éléments relevant du jeu. Par opposition au serious game, la gamification transforme un outil ou une tâche en y ajoutant des éléments issus de la conception du jeu, mais en se limitant à certains de ses constituants. Si l'ensemble est repensé, nous sommes plutôt dans le cas d'une conception de serious game (Detering et $\mathrm{al}, 2011$ ). Nous pouvons citer à titre d'exemples : l'ajout de graphismes ou de sons rappelant un jeu, l'attribution de points ou autres récompenses virtuelles (badges ou changement de classe du personnage de l'individu "joueur") à une personne pour la réalisation d'une tâche décomposés en étapes, la mise en compétition de personnes sous la forme de challenges ou de parties, l'affichage des meilleurs scores sur un espace commun ou leur diffusion via un réseau social, etc.

Le courant de la gamification n'a pas que des adeptes, il subit aussi de nombreuses critiques (Haydée, 2013 ; Silva, 2013). En effet, la mise en compétition de personnes sur la base de tâches de travail récompensées par l'attribution de points est souvent qualifiée de phénomène de "pointification". Réduite à cela, la gamification n'a rien de bien originale et ne peut bénéficier que peu de temps d'un attrait en tant que nouveauté (Schacht et al, 2014).

Il faut toutefois reconnaître certains succès à la gamification comme la solution de GPS Waze développée par une start-up israélienne. La présentation de l'interface du GPS sous une forme plus ludique avec des icônes rappelant certains jeux vidéo (figure 5) associée à des challenges et des points gagnés pour les automobilistes se déplaçant sur les lieux pointés par des icônes, a permis à cette entreprise de dresser des cartes plus précises et ajuster les itinéraires proposés (Marczewski, 2013, 15-16). Depuis, l'entreprise a été rachetée et désormais l'application Waze est détenue par Google et est plus utilisée qu'un autre logiciel américain : Coyotte. 


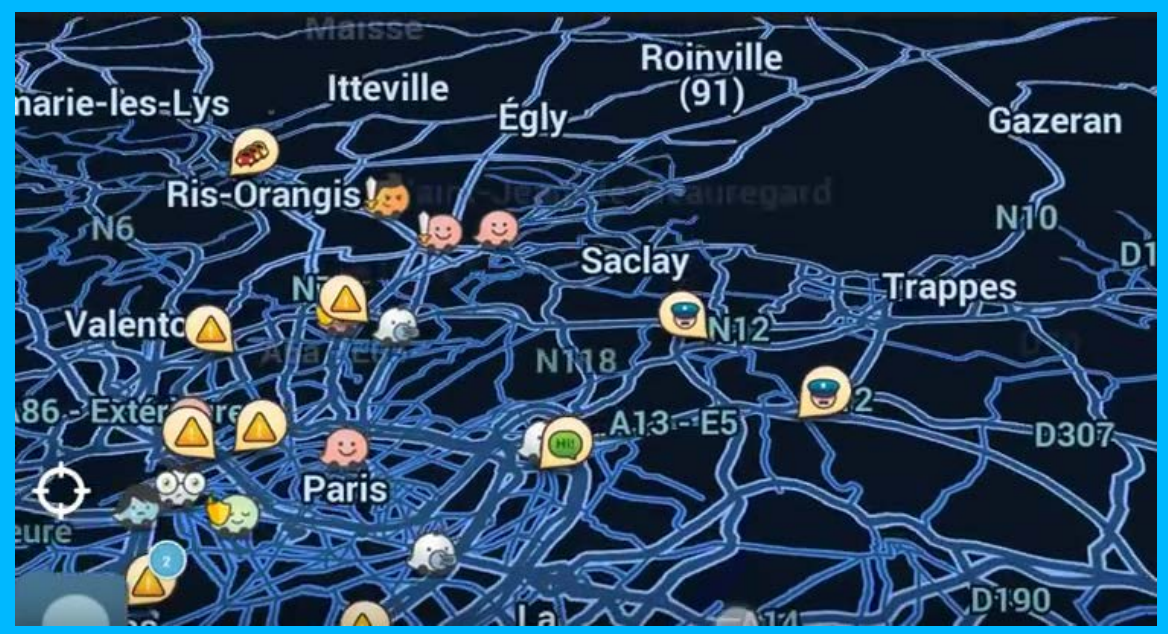

Figure 4. Waze

En termes de visualisation de données, une gamification est une solution qui répond à un problème posé de la manière suivante : « quelles transformations relevant du jeu ou rappelant le jeu peut-on réaliser à partir outils de présentation d'information existants pour intéresser plus de personnes ou solliciter plus d'attention auprès d'un panel de personnes ? ».

La réponse à cette question peut être trouvée en se référant à des solutions typiques d'expérience de gamification, mais aussi identifiant d'abord le public visé ainsi que ses perceptions, connaissances et préférences en termes de jeux. La gamification ne nécessite pas forcément de grands moyens financiers. Cependant, pour la mettre en œuvre, il faut être en mesure de pouvoir ajouter à un outil des éléments relevant du jeu. Si le problème est interprété d'un point de vue informatique, ceux sont donc des outils open source qui seront les plus adéquats pour subir ce type de transformation.

\section{Le disengamement}

Comme évoqué ci-dessus, les applications issues du jeu ou inspirées par le jeu sont de plus en plus nombreuses, mais en termes de catégories ne sont pas dénuées d'ambiguïtés. C'est en réfléchissant sur les distinctions entre serious game, game with a purpose, serious play et logiciel ayant suivi une gamification qu'il nous a semblé qu'une autre catégorie manquait : celle des jeux qui était déformés au point que l'application résultante ne puisse plus être qualifiée de jeu. Une fois cette catégorie établie, nous avons alors pu en trouver de nombreux exemples. Dans ce papier, nous nous concentrons sur la présentation du concept de disengamement et la mise en œuvre de ce dernier à des fins de cartographie.

\subsection{Présentation du concept}

Le terme «disengamement» est un néologisme qui tente d'exprimer l'idée de la transformation d'un jeu en quelque chose d'autre destinée à une fin sérieuse. Il s'agit d'un désengagement ludique (on ne joue plus vraiment) associé à une déformation de la structure du jeu initial. En fait, un jeu unique pleinement identifié comme tel est transformé en quelque chose 
Les visualisations de données inspirées par le jeu 9 qui n'est plus un jeu. Certains de ses éléments constitutifs qui touchent à sa nature même de jeu vont lui être retirés. Cette approche suit le processus inverse de celui de la gamification. Au départ, il y a bel et bien un jeu auquel on retire ou on ajoute des éléments qui vont faire que son adaptation à des fins sérieuses sera conforme aux attentes. Ces transformations vont induire dans le résultat final de tels changements qu'il ne pourra pas plus être véritablement qualifié de jeu, mais pourra encore le rappeler. A ce titre la décomposition de la structure d'un jeu en quatre facettes comme l'a proposé Jesse Schell $(2010,51)$ peut nous aider à identifier les éléments essentiels de la structure d'un jeu qui peuvent être modifiés ou préservés : son esthétique, ses mécanismes (procédures et règles), sa technologie (tous les matériels et matériaux sollicités par le jeu) et son histoire (l'ensemble des séquences d'évènements qui compose son déroulement).

Bien entendu tout jeu "disengamemé" peut être considéré comme une structure gamifiée, si l'on ne considère que le résultat final. De même tout jeu ayant subi un processus de disengamement, mais reste jouable peut être considéré, en fonction l'ampleur des modifications apportées au jeu d'origine, comme un serious play (peu de modifications) ou serious game (de nombreuses modifications qui en font une sorte de jeu à part). L'application NewsBlaster en est une illustration (figure 5). Du point de vue de la gamification, il s'agit d'une page web d'information à laquelle on a ajouté des éléments de jeu pour mieux intéresser certains lecteurs. Du point de vue des serious games, il s'agit d'une application sous la forme d'un jeu sérieux dont l'objectif utilitaire concerne l'affichage d'informations d'actualité. Du point de vue du disengamement, il s'agit du jeu vidéo Puzzle Bobble qui a été détourné et transformé afin d'atteindre un objectif sérieux tout en préservant l'essentiel de son esthétique et de ses mécanismes (Alvarez et Djaouti, 2012, 48-49). Le mécanisme de gain de points reste le même que dans le jeu original. Il s'agit d'assembler au moins quatre bulles de la même couleur. Lorsque c'est fait, les bulles explosent libérant de la place, des points sont alors obtenus et une nouvelle information d'actualité apparait.

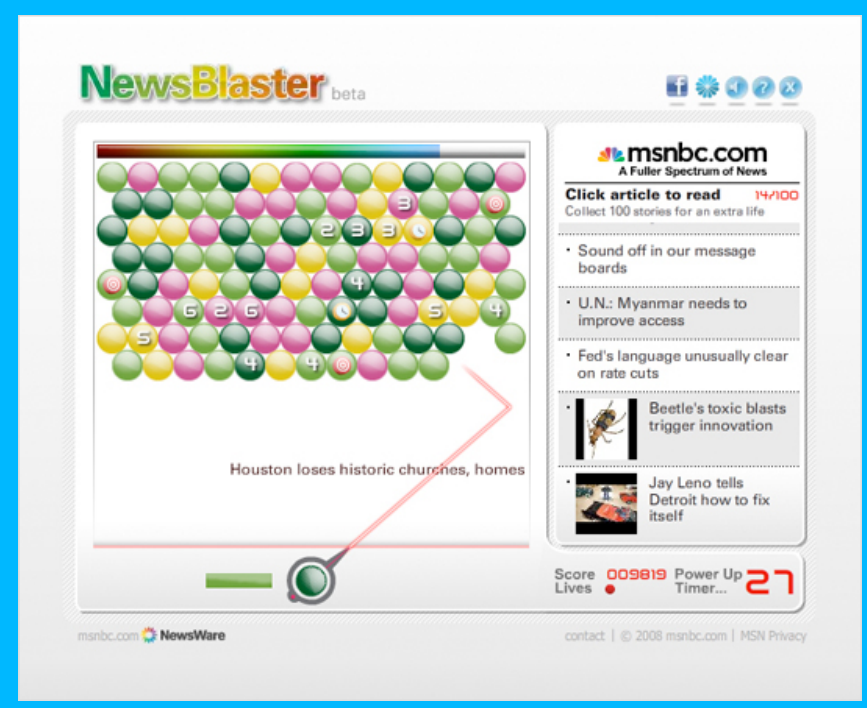

Figure 5. Légende figure sous la figure

Du point de vue de la visualisation de données, un disengamement réussi consiste en une solution qui répond à un problème posé de la manière suivante: "quelle présentation d'information sous la forme d'une photographie d'une partie de jeu à un instant $t$ mettrait en 
valeur, mieux que d'autres graphiques, des informations pertinentes pour mon problème ?». De cette question découle donc à la fois de l'identification d'un besoin cartographique auquel on souhaite répondre, de la reconnaissance d'un jeu particulier pouvant s'y conformer ainsi que de l'instant de la partie à "photographier".

La réponse à cette question peut être trouvée en prenant connaissance de nombreux jeux, ce qui peut inclure la prise en compte d'une connaissance de leurs mécanismes. Elle peut aussi être formulée en utilisant un système de traduction de besoins graphiques en éléments caractéristiques. L'interrogation d'une base de données de jeux définis selon ces caractéristiques permettra d'identifier un nombre réduit de jeux qui pourront être adaptés pour résoudre ce problème. Comme dans les autres cas d'emplois du jeu à des fins sérieuses cités ci-dessus, il sera aussi nécessaire de prendre en compte le public visé par la future lecture de la cartographie obtenue. Puisqu'elle rappellera encore le jeu dont elle sera issue, le jeu choisi doit être connu ou facilement compréhensible par les personnes à qui la cartographie sera présentée. Mieux, si le jeu n'est pas trop dénaturé en termes de mécanismes de mise en forme, alors certains publics pourront s'approprier cette cartographie s'ils sont familiers du jeu originel et l'apprécient.

\subsection{Cas de disengamement d'un jeu à des fins de cartographie}

Afin d'illustrer la mise en œuvre d'un procédé de disengamement, nous proposons de cartographier le marché de l'automobile pour 15 pays européens et un ensemble de constructeurs. En fait, tous les ans en août, les statistiques de ce marché sont librement disponibles sous la forme d'une série de tableaux statistiques commentés ${ }^{1}$, ce qui nous donne l'opportunité d'envisager une visualisation de données d'un univers concurrentiel. De plus, en parcourant ces rapports, il nous a semblé que les divers tableaux et diagrammes en lignes ou en barres n'étaient pas forcément suffisants pour représenter l'évolution de ce marché. Dans le cadre de cet exemple, nous avons limité ce travail de mise au cas des immatriculations de voitures particulières neuves par pays et par groupe en Europe.

Afin de disposer d'assez de données statistiques, nous avons retenu les pays pour lesquels nous avions toutes les statistiques depuis 2002, soit les 15 pays suivants : Allemagne, Belgique, Danemark, Espagne, Finlande, France, Grèce, Irlande, Luxembourg, Norvège, Pays Bas, Portugal, Royaume Uni, Suède et Suisse. Vis-à-vis de ces 15 pays, nous avons pu disposer des données de 10 groupes constructeurs automobiles: Groupe PSA Citroën, Groupe Renault, Groupe Fiat, Groupe Volkswagen, Groupe Ford, groupe General Motors, groupe BMW Mini, Groupe Daimler, Marques japonaises, Marques coréennes.

\subsubsection{Choix du jeu}

Comme définie plus haut, la mise en œuvre d'un processus de disengamement commence par une question. Dans le cas de notre exemple, elle pourrait prendre la forme suivante: "quelle présentation d'information sous la forme d'une photographie d'une partie de jeu à un instant $\mathrm{t}$ mettrait en valeur le marché de l'automobile en Europe ?». Cette question en entraîne une autre : « quel jeu existant permettrait de représenter l'affrontement concurrentiel 10 groupes de constructeurs sur un terrain correspondant à 15 pays européens ? ». Une autre question importante concerne aussi l'identification du public auquel est destinée la cartographie désirée.

${ }^{1}$ Par exemple $:$ http://www.ccfa.fr/Edition-2014 
Les visualisations de données inspirées par le jeu 11 Nous proposons de choisir un public assez large, ce qui nous ajoute la contrainte supplémentaire que le jeu recherché doit lui aussi être grand public, ce qui peut se traduire par le fait que le jeu doit être si possible très connu et ce qui est souvent lié avoir des règles simples. Ensuite, nous pouvons revenir sur le besoin informationnel : mieux comprendre l'évolution et la répartition des constructeurs automobiles sur le marché européen. Ceci revient à devoir montrer les effets de la concurrence sur ce marché à partir des dix groupes de constructeurs automobiles dont nous avons les statistiques. Cela sous-entend aussi que les rapports de forces entre les différents groupes automobiles ne doivent pas être équilibrés et donc que certains peuvent se développer au détriment d'autres.

Il faut donc identifier un jeu à plusieurs joueurs (au moins 4 que l'on étendrait à 10) mettant en évidence des affrontements et des déséquilibres de forces qui si s'appuierait sur un système de protection et de conquête de territoires que l'on pourrait lier aux différents pays d'Europe. Ceci peut se traduire facilement en termes de jeu : un jeu compétitif multijoueur dont le plateau représente plusieurs zones géographiques. Une fois cela fait, nous pouvons faire le bilan des jeux que nous connaissons ou bien utiliser un site ou une base de données dédié aux jeux. Par exemple, le site boardgamegeek consacré aux jeux de plateau², avec son mode de recherche avancée, peut être une source d'information très intéressante lorsque nous cherchons un jeu aux caractéristiques particulières ${ }^{3}$. Parmi les filtres proposés, nous pouvons utiliser le filtre "sous domaine de jeu » avec le critère « jeu familial» (Family Games) afin de trouver un jeu grand public et nous choisissons aussi le filtre "mécanique de jeu» avec les critères "aires de contrôle / d'influence » (Area Control / Area Influence) et « aires de mouvements » afin d'obtenir un jeu fondé sur le principe du contrôle de territoires. Nous employons en plus de ces filtres, le filtre destiné à ne pas afficher les extensions (Click to NOT search expansions) des jeux. Nous obtenons avec cette requête 104 résultats.

À partir de ces résultats, nous pouvons parcourir les descriptions et photographies des jeux. Cela prendra environ 6 heures à raison de 3 minutes destinées à ce parcours ainsi qu'à une prise de notes visant l'élimination ou la sélection d'une dizaine de jeu. 2 ou 3 heures d'études plus approfondies devraient nous permettre de sélectionner 1 à 3 jeux. Ce processus est un peu long, mais est envisageable puisqu'il représente environ une journée de travail. Une alternative plus rapide, consiste à choisir les 6 à 12 jeux ayant le meilleur classement de la sélection (les meilleures appréciations) avant de les étudier avec attention durant 2 à 3 heures. Une autre solution, très pratique, peut consister en l'ajout d'un critère de sélection. Par exemple, le filtre "mécanique de jeu» avec le critère "aires de mouvements» (Area Movement) permet de rechercher des jeux qui prennent en compte des mouvements. Nous obtenons alors une liste 14 jeux. Il nous faudra environ 3 à 4 heures pour explorer ce panel réduit et sélectionner 1 à 3 jeux.

Dans notre cas d'exemple, même si plusieurs jeux semblent intéressants, le critère de choix d'un jeu connu nous incite à réduire notre sélection à des jeux pour lequel de très nombreuses personnes ont voté et donc joué ("Num Voters») un jeu comme Small Word ou le Risk. Comme nous connaissons bien le Risk et très peu Small Word, nous choisissons de tenter l'expérience de disengamement avec le Risk, ce sera bien plus rapide. Dans le cas contraire, il nous aurait fallu nous familiariser avec le jeu Small Word en nous le procurant et si possible en y jouant.

\footnotetext{
${ }^{2}$ Deux sites équivalents dédiés aux jeux de rôles (rpggeek) et aux jeux vidéo (videogamegeek) existent par ailleurs.

${ }^{3}$ Nous aurions pu faire une recherche équivalente sur une base de données de jeu un peu moins importante, mais francophone ; par exemple, celle du site Tric Trac. Cependant le nombre de jeux et de critères de recherche disponible sur ce site, nous incite à préférer le site boardgamegeek.
} 


\subsubsection{Présentation du jeu sélectionné : le Risk}

Il s'agit, pour sa version la plus connue, d'un jeu guerre territorial pour 2 à 6 joueurs dont le plateau représente une carte du monde où les continents sont divisés en territoires. Il s'agit d'un jeu d'affrontements dans lequel chaque joueur dispose de pions d'une couleur qui lui est propre et servant à distinguer ses armées de celles des autres. En début de partie, chaque joueur dispose d'un nombre déterminé de territoires et de troupes qu'il répartit comme bon lui semble sur chacun d'eux. Au début du jeu, chacun des adversaires dispose d'autant de territoires et de troupes que les autres, mais ce nombre varie ensuite au grès des affrontements et des ravitaillements. A ces fins, le jeu utilise deux jeux de cartes. L'un permet de déterminer à l'aide d'une carte, l'objectif que l'on doit remplir pour gagner la partie. L'autre est utilisé tout le long de la partie pour tirer au sort des ressources militaires à chaque conquête effectuée. Pour gagner en place et en lisibilité sur le plateau, les unités du Risk sont représentées par trois catégories de pions aux formes distinctes afin de présenter à l'aide d'un seul pion : 1 unité, 5 unités ou 10 unités.

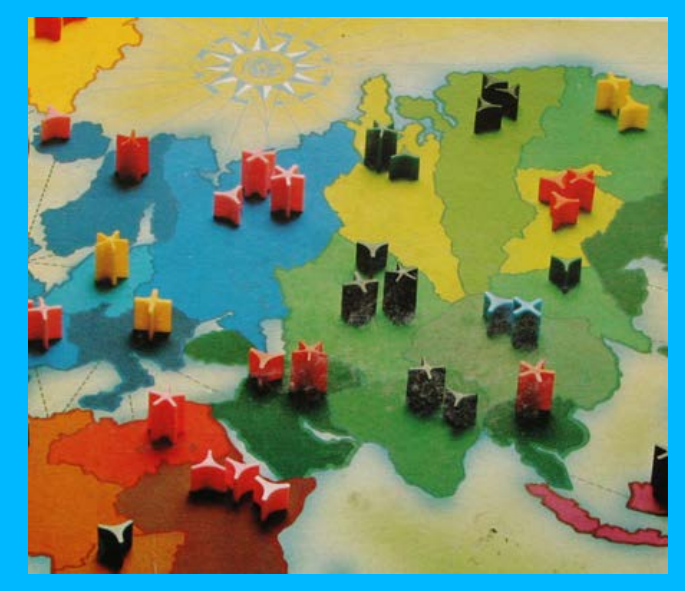

Figure 6 : Exemple de placements de pions lors d'une partie de RISK (édition standard)

Du point de vue des mécanismes de jeu, le Risk est un jeu qui se joue « au tour par tour ». Cela signifie que chacun des joueurs attend son tour pour jouer, sauf s'il est attaqué par un autre joueur. Ainsi, lorsque vient son tour, un joueur peut choisir un ou plusieurs territoires qu'il souhaite attaquer (soient des territoires contigus à un territoire d'où va partir l'attaque, soient reliés à ce dernier par des pointillés). Pour ce faire, il doit disposer d'au moins 2 unités sur un territoire adjacent à ceux qu'il prend pour cible. Les affrontements sont alors simulés par une série de jets de dés qui prend fin lorsque le joueur attaquant le décide ou élimine toutes les troupes qui se trouvaient sur le territoire attaqué. S'il gagne l'affrontement, le joueur doit alors placer au moins 1 unité sur le territoire conquis et laisser au moins 1 unité sur le territoire duquel est partie l'armée d'invasion. Il reçoit alors une carte ravitaillement (en unités) qu'il pourra employer lorsqu'il le souhaitera.

\subsubsection{Critères de transformation analogiques retenus}


Les visualisations de données inspirées par le jeu 13

Notre objectif principal est d'ordre cartographique et chronologique, il s'agit de proposer, sur la base du support de jeu Risk, une cartographie de la situation du marché de l'automobile en Europe qui changera d'année en année. Il s'agissait de représenter 15 pays européens et 10 groupes de constructeurs automobiles différents. Afin de transformer le jeu Risk en une cartographie des constructeurs automobiles pour les pays européens concernés il nous faut faire un point sur ce qui est possible tel que et ce qui va nécessiter un changement ou un compromis.

Comme le Risk est un jeu de guerre impliquant des contrôles de zones territoriales, nous gardons cet aspect esthétique du jeu. Nous l'avions recherché et sélectionné pour cette caractéristique. Pour ce qui concerne le nombre de groupes automobiles à représenter, si au Risk le nombre maximum d'armées différentes représentées est de 6 (une par joueur), il nous faut, en suivant le même principe, ajouter d'autres joueurs (un joueur correspond à un constructeur automobile) en leur associant de nouvelles couleurs (figure 7). Cet ajout ne change pas la mécanique du jeu, seulement son esthétique. De même, les écarts de chiffres présentés dans les tableaux statistiques qui nous servent de données nous incitent à distinguer le nombre d'unités présentes sur un territoire à l'aide 4 catégories de pions plutôt que 3 (figure 7).

Du point de vue, du jeu, les éléments les plus simples à modifier ou à adapter ont été passés en revue. Il nous faut maintenant nous préoccuper des changements plus profonds à opérer pour transformer les données des statistiques en une présentation qui rappelle une partie de Risk. Pour cela, il nous faut ainsi rendre compte de divers éléments

\begin{tabular}{|l|c|l|c|}
\hline PSA Peugeot Citroën & $\square$ & General Motors & \\
\hline Groupe Renault & $\square$ & BMW-Mini & \\
\hline Groupe Fiat & & Daimler & \\
\hline Groupe Volkswagen & & Marques japonaises & \\
\hline Groupe Ford & & Marques coréennes & \\
\hline$\bigcirc \quad=$ mille unités & $\triangle=5$ milles unités & $\square=25$ milles unités & $\square=100$ milles unités \\
\hline
\end{tabular}

Figure 7 : Légende des couleurs et symboles employés pour représenter à la manière du Risk le marché européen de l'automobile

Au Risk, une partie se décompose en tours de jeu eux-mêmes constitués de 3 phases d'action (ravitaillements, attaques et mouvements) que peut exploiter à son tour chacun des joueurs. Un tour complet correspond au fait que tous les participants à la partie ont pu jouer durant le même tour. La version disengamemée du Risk présentera une série de cartographies ressemblant à la situation d'une partie de Risk à la fin d'un tour complet. Un tour correspondra à une année d'immatriculations, car il s'agit de la périodicité des données dont nous disposons.

Au Risk, il ne peut y avoir qu'une seule armée sur un même territoire. Dans notre version disengamemée, un seul constructeur ne pourra occuper qu'une seule zone territoriale.

Au Risk un pays est découpé en une à plusieurs zones territoriales dont le nombre représente son importance. Dans notre version disengamemée, les pays devront être découpés en territoires selon leur importance. Comme au Risk la taille d'un territoire n'a pas d'influence sur le nombre d'unités pouvant y figurer, sur la cartographie les pays pourront être découpés en territoires de surface à peu près équivalente. La taille d'un pays pourra être associée à sa part de marché vis-à-vis du nombre d'immatriculations total des 15 pays européens sélectionnés. Le nombre de territoires composant un pays dépendra de la taille minimale choisie en termes 
d'immatriculations pour qu'un pays soit composé d'au moins 2 territoires. Sous un certain seuil, les plus petits pays, en termes d'immatriculations, seront représentés par un seul territoire.

$\mathrm{Au}$ Risk une armée ne peut attaquer une autre zone géographique, seulement si le territoire attaqué est contigu au sien ou lié par des pointillés illustrant une voie maritime entre les 2 territoires. Donc, dans notre version disengamemée, un constructeur possédant des unités sur un territoire pourra être perçu comme pouvant potentiellement attaquer des territoires proches (adjacents ou séparés par un petit bras de mer) ou subir une attaque par un autre constructeur de l'un de ces territoires. Il découle de cela, le fait que les proximités des territoires dessinés sur la carte devront traduire une proximité ou un éloignement territorial. Les territoires doivent pouvoir être estimés les uns par rapport aux autres en termes de distance liée à leurs immatriculations automobiles

Au Risk, ce sont les armées les plus fortes qui attaquent les plus faibles. Dans la version disengamemée, il faudra rendre compte des faiblesses et des forces des constructeurs sur certains territoires. Pour ce faire, nous proposons de ne représenter que la différence d'immatriculations d'un constructeur à un autre sur un pays donné en fonction du nombre d'immatriculations du plus important constructeur non représenté sur le pays. De la sorte, pour pays constitué d'un seul territoire, si deux groupes de constructeurs ont un nombre d'immatriculations nouvelles quasi identique et qu'il s'agit du plus grand nombre d'immatriculations nouvelles pour ce pays, alors ce pays ne sera occupé par aucun groupe 4 .

\subsubsection{Constitution d'une cartographie sous la forme d'un plateau de Risk}

Une fois identifiées les transformations par analogies qui pouvaient être faites, il nous faut passer à l'application. Afin de déterminer la taille des marchés et donc définir celle des territoires, nous avons regardé pour chaque pays la valeur maximale de son total d'immatriculations nouvelles entre 2000 et 2013. Nous avons ainsi obtenu deux catégories de pays en termes de marché. Le premier groupe de pays rassemble les pays dont la taille maximale du marché est comprise entre $1600 \mathrm{mni}^{5}$ et $3800 \mathrm{mni}$ : l'Allemagne (valeur maximale : 3802 mni), le Royaume-Uni (valeur maximale : $2476 \mathrm{mni}$ ), l'Italie (valeur maximale : $2460 \mathrm{mni}$ ), la France (valeur maximale : $2291 \mathrm{mni}$ ) et l'Espagne (valeur maximale : $1628 \mathrm{mni}$ ). Le second groupe de pays rassemble les pays dont la taille maximale du marché est comprise entre 700 mni et 50 mni. Le plus important est celui des Pays-Bas avec une valeur maximale de $661 \mathrm{mni}$ et le plus petit est celui du Luxembourg avec une valeur maximale de $52 \mathrm{mni}$. De manière très simple, nous avons divisé le plus grand pays en 10 territoires, en nous laissant une petite marge d'erreur pour le plus grand pays (l'Allemagne), nous avons alors considéré qu'un territoire correspondrait à 410 mni 6 .

Ensuite, il nous a fallu redéfinir la proximité entre les pays et, par voie de conséquence, aussi leur forme. Pour définir cette proximité, il nous a semblé nécessaire d'utiliser des statistiques relatives au marché de l'automobile en Europe pour réinterpréter les distances entre les différents pays, mais aussi des statistiques différentes de celles employées pour représenter la

\footnotetext{
${ }^{4}$ C'est le cas de la Finlande en 2010 (figure 11 : dans le coin inférieur droit). Nous pouvons aussi remarquer sur cette carte que la Finlande possède une frontière commune avec la Norvège, mais surtout avec le Portugal.

${ }^{5} \mathrm{Mni}$ : milliers de nouvelles immatriculations

${ }^{6}$ Le nombre de territoires d'un pays correspond donc au résultat de la division de son nombre maximum d'immatriculations entre 2000 et 2013 divisé par 410 mni, le tout arrondi à l'entier supérieur. Un pays comme les Pays-Bas correspond donc à 2 territoires puis que $661 / 410$ donne environ 1.61 .
} 
Les visualisations de données inspirées par le jeu 15 force des constructeurs sur les différents pays européens. Nous avons alors exploité les tableaux statistiques des rapports de 2000 à 2013 des 17 pays pour les ventes pour 6 catégories de véhicules : Berlines, Breaks, Coupés, Cabriolets, Monospaces, Autres. Pour chacune des années, nous avons d'abord traduit en pourcentage ce que chaque catégorie de véhicules représentait pour le pays considéré. Nous avons ensuite calculé, catégorie par catégorie, la valeur absolue de la différence de vente entre les pays pris 2 par 2. Pour une année, nous avons ensuite fait pour chaque paire de pays la moyenne pour l'ensemble des catégories ce à quoi nous avons ajouté et de l'écart-type qui correspondait. Puis, nous avons fait un tableau faisant la somme des moyennes et des écarts types obtenus par paire de pays pour toutes les années de 2000 à 2013. Nous avons obtenu ainsi un chiffre représentant pour deux pays donnés une valeur exprimant une certaine distance existante entre eux. Il ne restait qu'à définir un seuil à dépasser pour que deux pays soient séparés par de l'eau, c'est-à-dire "seuil de mer". Enfin, nous avons réutilisé notre tableau des tailles maximum de marché par pays pour définir la taille du territoire en prenant pour repère le plus petit pays qui est le Luxembourg, mais que nous souhaitions distinguer sur la cartographie obtenue.

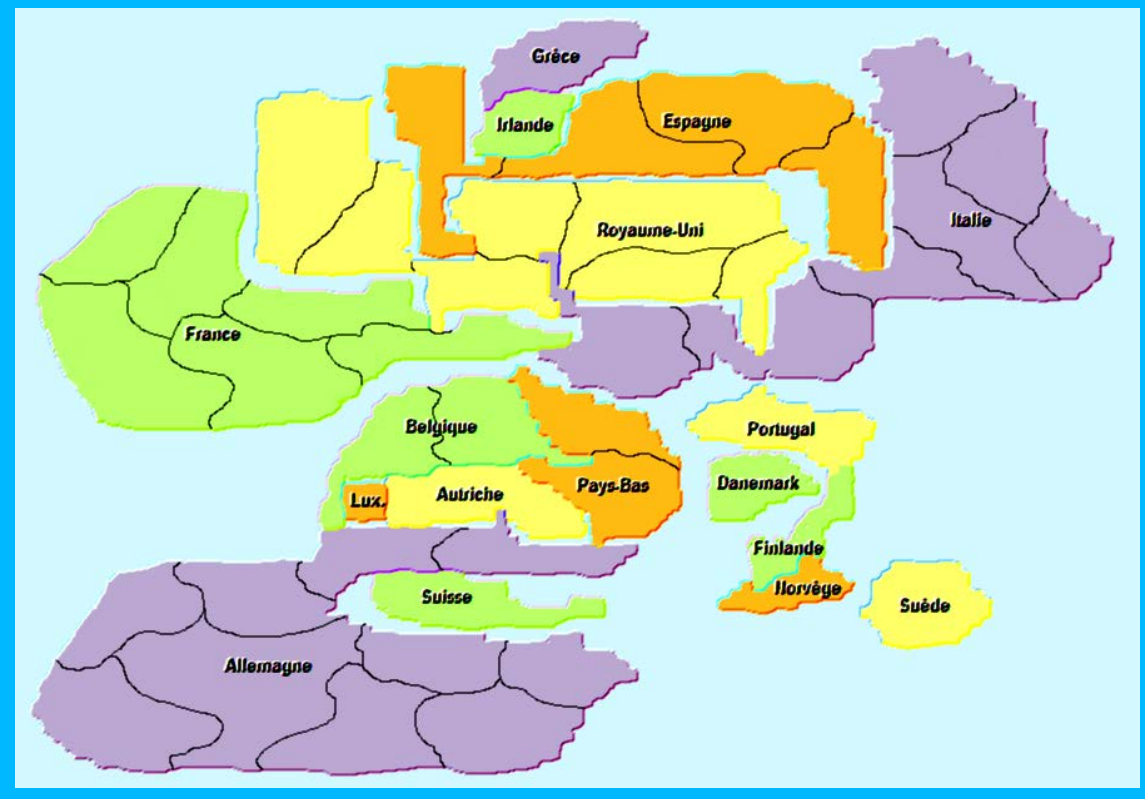

Figure 8 : carte vierge représentant les distances entre les pays européens et leur nombre de divisions en parts de marché

En utilisant un logiciel de traitement graphique, nous réalisé un quadrillage qui nous permettait d'associer 4 pixels à $1 \mathrm{mni}$ de la valeur maximale de la taille d'un marché et ainsi avoir une base pour représenter tous les pays. Dans une première étape, nous avons réalisé des formes approchant le carré, puis nous avons utilisé la distance estimée entre les pays et le "seuil de mer" pour placer les pays les uns par rapport aux autres en commençant par les plus importants. Un avantage de ce type de représentation par rapport celle produite par une analyse en composantes principales, par exemple, est que nous pouvons changer la forme d'un pays pour qu'il soit à la fois proche d'un pays et loin d'un autre.

Nous avons aussi, pour améliorer l'esthétique des pays, déformé un peu plus les bases rectangulaires que nous avions utilisées au départ pour les tracer en déplaçant une nouvelle fois 
les pixels utilisés pour les tracer en évitant de modifier la distance séparant les pays. Nous avons ainsi rendu plus naturelle la forme des pays de la carte. Nous avons aussi ajouté un effet "relief" aux pays afin de, plus tard, mieux distinguer les pions des territoires sur lesquels ils seraient placés.

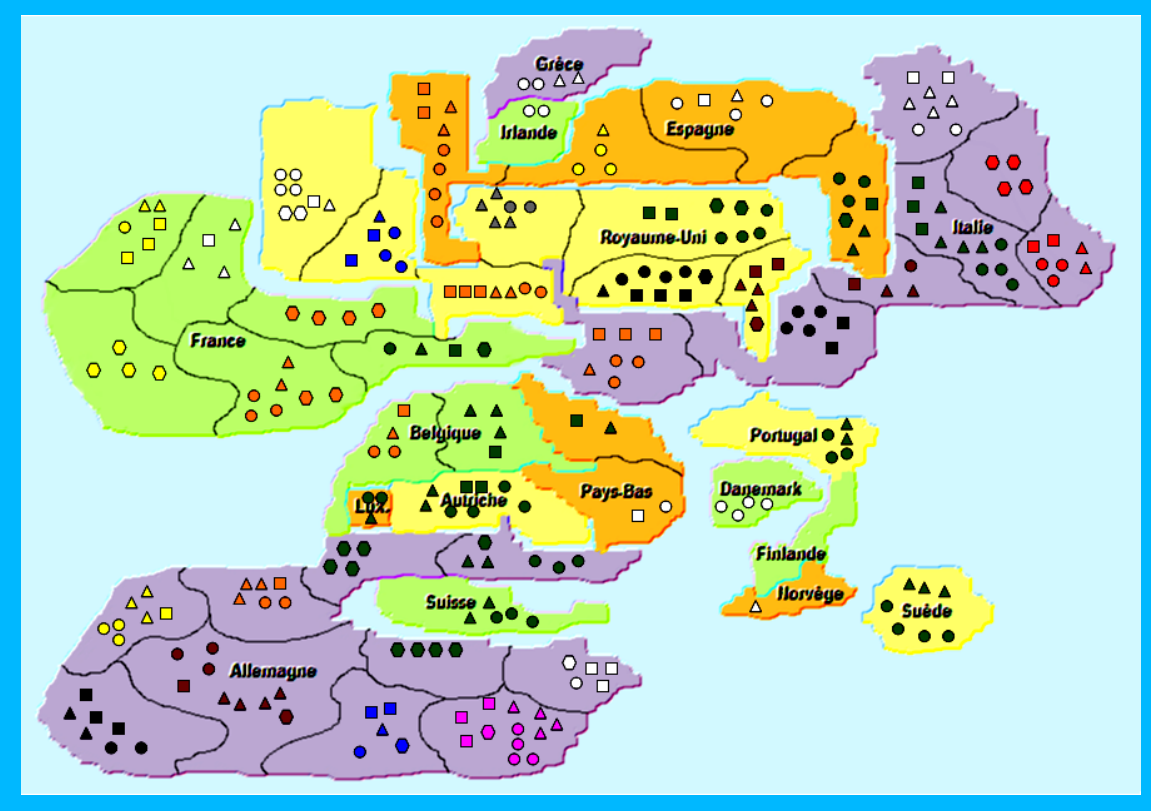

Figure 9 : Représentation du marché de l'automobile en Europe en 2010

Sur le modèle du jeu Risk, avec ce système de représentation la taille de la frontière commune entre deux pays ne compte pas ; ce qui compte c'est qu'il y ait une frontière commune ou une forte proximité, même entre deux petits bras de terre, pour que l'on puisse envisager de passer d'un pays vers un autre. C'est ainsi que nous avons obtenu la figure 4 pour laquelle nous avons agrandi le nom des pays pour mieux les identifier. Puis, pour chaque année, nous avons placé sur les territoires du plateau (figure 8) les unités de chaque groupe de constructeurs automobiles. Ce nombre d'unités est défini en fonction du nombre de territoires disponibles pour chaque pays, le classement d'un groupe de constructeurs dans la hiérarchie des groupes qui vendent le mieux sur ce pays et, arrondi à l'entier supérieur, le résultat de soustraction entre les ventes du constructeur considéré en termes de mni pour ce pays et le nombre de mni du groupe ayant les meilleurs résultats, mais dont la position hiérarchique se situe en dessous du nombre de territoires du pays, le tout divisé par $410 \mathrm{mni}$ (taille maximale d'un territoire). Les unités de ce groupe sont alors réparties de façon homogène sur l'ensemble des territoires qu'il occupe sur ce pays.

Ensuite, nous avons tenté de représenter une certaine cohérence dans le placement des unités d'un même constructeur. Ainsi ; dans la mesure du possible, les unités d'un même groupe qui sont placées sur des territoires de pays différents, mais très proches, voire limitrophes sont mis sur les territoires qui rendent le mieux compte de cette proximité (figures). Nous avons aussi utilisé quatre différentes catégories de pions pour réduire le nombre de pions affichés sur chaque carte: les cercle représentent $1 \mathrm{mni}$, les triangles $5 \mathrm{mni}$, les carrés $25 \mathrm{mni}$ et les hexagones $100 \mathrm{mni}$. 
Les visualisations de données inspirées par le jeu 17

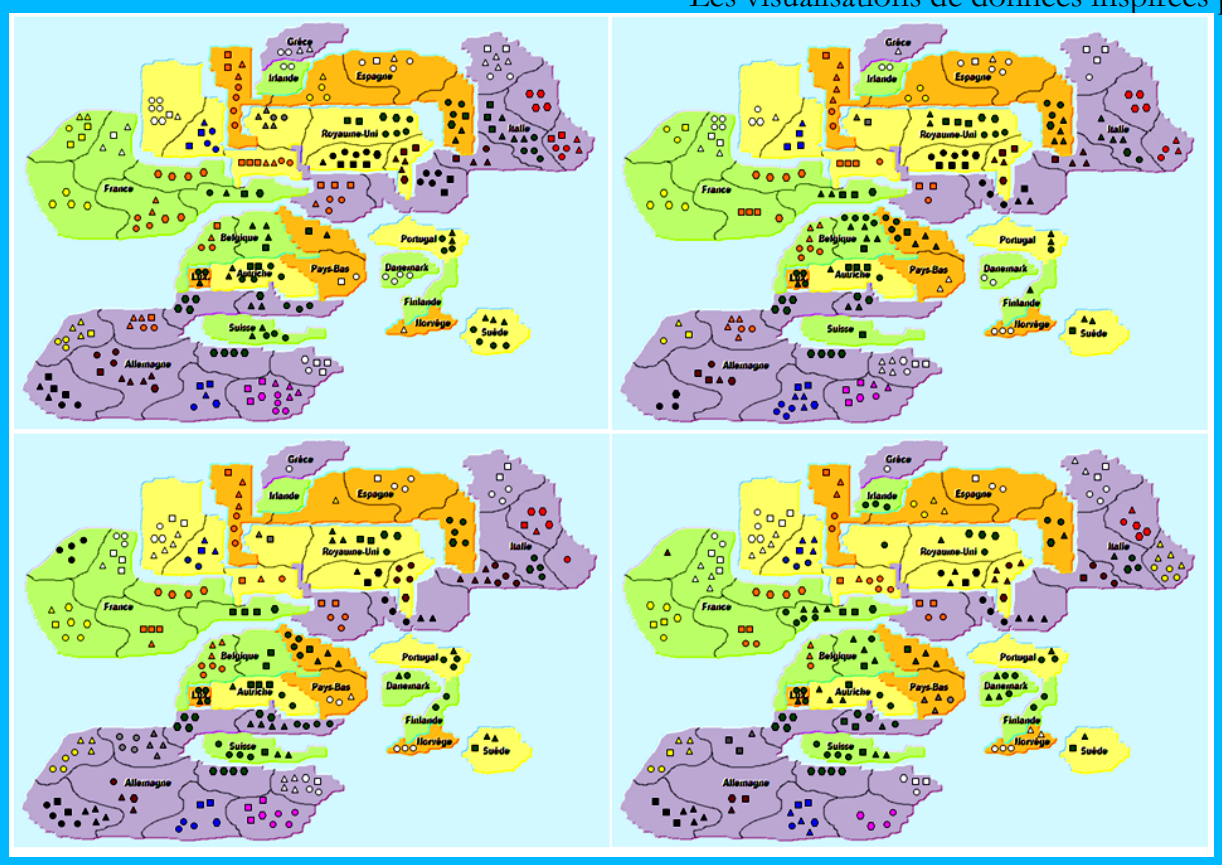

Figure 10 : Cartographies du marché de l'automobile en Europe sous la forme d'une partie de Risk entre 2010 (en haut à gauche) et 2013 (en bas à droite)7.

Au final, nous disposons de plusieurs cartes (figure 10) qui peuvent se lire, chacune, comme une photographie de fin de tour d'une partie de Risk. Cela donne un effet dynamique et aussi peut aider à raconter l'évolution du marché. Bien entendu, certains compromis qui ont été faits peuvent dérouter le lecteur de la carte. Ainsi, certaines apparitions de groupe sur un pays peuvent donner l'impression d'une sorte de parachutage. En effet certaines "conquêtes" semblent très loin d'un territoire où d'autres unités du même groupe sont présentes. En fait, l'occupation historique (à l'échelle de la partie représentée) fait que certaines nouvelles occupations sur des territoires proches ne sont pas possibles, car elles impliqueraient un déplacement de nombreux groupes et donc une reconfiguration importante de la carte. Cela irait à l'encontre du principe d'inspiration d'une partie de jeu. A l'inverse, nous pouvons envisager que ce phénomène est dû à une modification des règles du jeu originel. C'est aussi en ce sens que le jeu Risk est déformé. Ces règles ne sont plus exactement les mêmes et ce qui est présenté au final est bien plus une cartographie qu'un jeu.

\section{Premiers retours d'expérience et conclusion}

Après avoir réalisé ces cartographies, nous les avons utilisées et présentées sous forme numérique, d"impressions papier et sous forme de jeu. Actuellement, les quelques personnes à qui nous les avons présentés sont des enseignants qui s'intéressent au jeu, des consultants pour qui la mise en forme d'information stratégique est importante, des étudiants en sciences de

\footnotetext{
${ }^{7}$ On peut observer par exemple la disparition progressive du groupe Fiat (rouge) dont les parts en Italie sont grignotées petit à petit, la diminution du même ordre des unités de Daimler (rose) en Allemagne, ainsi que la prise de contrôle progressive du centre de la carte du groupe Volkswagen.
} 
l'information et de la communication en Master 1 et en sciences de gestion en Master 2, ainsi que quelques personnes curieuses lors de deux soirées grands publiques d'illustrations d'application originales en rapport avec le jeu.

Les premiers retours d'expérience que nous en avons tirés concernent à la fois l'intérêt des personnes pour les cartographies proposées et leur compréhension du marché de l'automobile à partir de celles-ci. Il s'agit uniquement d'observation de réactions face aux cartographies, suivi en cas d'intérêt, de quelques questions à propos de leurs connaissances des jeux de plateau ainsi que sur ce qu'ils comprenaient de ce qui étaient présenté. Ainsi, il semble que si le public à qui on présente les cartographies du marché de l'automobile sous la forme d'une partie de Risk disengamemée apprécie les jeux sur plateau, alors ce genre de cartographie est un très bon support pour la médiation d'informations stratégiques de ce type. Un autre élément qui nous semble intéressant concerne l'affichage papier ou numérique par projection sur un mur qui, pour être bien perçue par le grand public, doit indiquer clairement et amplement le titre le jeu d'origine qui est transformé. Si tel n'est pas le cas, les personnes défilent sans trop regarder les cartes ou bien comme ce fut le cas, peuvent demander s'il ne s'agit pas d'une forme d'art. Un autre résultat que nous avons constaté, lors d'une seule occasion, est la présentation d'une cartographie sous la forme de jeu. Le plateau a été imprimé au format A2 et avons placé des pions empruntés à d'autres jeux pour figurer les placements des groupes automobiles, le tout se trouvant non plus sur un mur, mais une table. Il semble que dans ce cas l'effet produit est plus important si plus d'une personne se trouve autour de la table et considère le "jeu" ou mieux manipule des pions. Les personnes familiarisés avec les jeux de stratégie sur plateau, qu'ils soient étudiants, enseignants ou consultants, semblent bien appréciés ce type de présentation tactile. Cependant, la demande de manipulation est alors très importante, voire un peu gênante, car toutes les personnes ne demandent pas l'autorisation de manipuler les pions. Il est alors difficile de garder une cohérence dans les placements des pions, ce qui est préjudiciable puisque la justification de la création de ce genre de visualisation peut relever d'un besoin médiation d'informations stratégiques. Si l'on change les pions de place sans raisonnement approprié, alors le message véhiculé ne peut être que faux. Ceci dit, par rapport au format numérique, les personnes semblent avoir bien plus envie de jouer à la place du constructeur le coup suivant. Une alternative que nous n'avons pas encore pu tester pourrait dès lors consister en l'emploi d'une table numérique tactile. Au final, il nous faut encore explorer d'autres formes de disengamement possibles tout en approfondissant les résultats encourageants déjà obtenus. De même les autres trois formes de visualisation de données inspirées par le jeu méritent d'être comparées à différentes formes de disengamement pour mieux cerner quand telle forme est plus adaptée que telle autre.

\section{Bibliographie non numérotée et références}

Abt C. (1970). Serious Games, University Press Of America, New York.

Alvarez J. et Djaouti D. (2012). Introduction au Serious Game, Questions Théoriques, Quercy.

Berget B. (2015). Yu Suzuki Le maître de SEGA : De l'arcade à la révolution Shenmue, Geeks-line.

Berry V. (2011). Jouer pour apprendre: est-ce bien sérieux? Réflexions théoriques sur les relations entre jeu (vidéo) et apprentissage. Canadian Journal of Learning and Technology/La revue canadienne de l'apprentissage et de la technologie, vol. 2, $\mathrm{n}^{\circ} 37$, p. 1-14. 
Les visualisations de données inspirées par le jeu 19

Brougère G. (2010). Formes ludiques et formes éducatives, Jeu et apprentissage: quelles relations, J. Bédard et G. Brougère (dir.), Sherbrooke, Editions du CRP, p. 43-62.

Caillois R. (1958). Les jeux et les hommes, Gallimard, Paris.

Cooper S., Khatib F., Treuille A., Barbero J., Lee J., Beenen M., ... \& Popović Z. (2010). Predicting protein structures with a multiplayer online game, Nature, Vol. 466, n 7307, p. 756-760.

Crookall, D. (2010). Serious Games, Debriefing, and Simulation/Gaming as a Discipline, Simulation \& Gaming, Vol. 41, n6, p. 898-920.

Deterding S., Dixon D., Khaled R. and Lennart N. (2011). From Game Design Elements to Gamefulness: Defining "Gamification", Proceedings of the 15th International Academic MindTrek Conference: Envisioning Future Media Environments, ACM New York, p. 9-15.

Frasca G. (2006). Playing with Fire: The Little Game That Could. Serious Games Source. http://seriousgamessource.com/features/feature_101806_little_game_1.php

Frick E., Tardini S. \& Cantoni L. (2013). White paper on LEGO® SERIOUS PLAY®: A state of the art of its applications in Europe, S-PLA: LEGO SERIOUS PLAY Learning for SMEs.

Grietzer F. L., Kuchar O. A. \& Huston K. (2007). Cognitive Science Implications for Enhancing Training Effectiveness in a Serious Gaming Context, ACM Journal of Education Resources in Computing, Vol. 7, $\mathrm{n}^{\circ}$ 3 , Article 2.

Haydée S. (2013). La « gamification » de la vie : sous couleur de jouer?, Sciences du jeu, n 1.

Huizinga J. (1951), Homo ludens : Essai sur la fonction sociale du jeu, Gallimard, Paris.

Henriot J. (1969). Le jeu, Paris, Presses universitaires de France.

Lafourcade M., Le Brun N. et Joubert A. (2015). Jeux et intelligence collective-résolution de problèmes et acquisition de données sur le Web, ISTE éditions.

Marczewski, A. (2013). Gamification: a simple introduction: Tips, Advice and thoughts on gamification.

Muletier C., Bertholet G. et Lang T. (2014), La gamification: ou l'art d'utiliser les mécaniques du jeu dans votre business, Editions Eyrolles, Paris.

Schacht S., Morana S., \& Mädche A. (2014). The project world-Gamification in project knowledge management, Proceedings of the European Conference on Information Systems (ECIS) 2014, Tel Aviv, Israel.

Schell J. (2010). L'Art du game design: 100 objectifs pour mieux concevoir vos jeux, Paris, PearsonEducation France.

Silva, H. (2013). La « gamification » de la vie: sous couleur de jouer?, Sciences du jeu, n 1.

Smith R. D. (2009). Military Simulation \& Serious Games: where we came from and where we are going, Modelbenders Press.

Von Ahn L. \& Dabbish L. (2008). Designing games with a purpose, Communications of the ACM, vol. 51, $\mathrm{n}^{\circ}$ 8, p. 58-67.

Weitze, C. L. (2014). Developing Goals and Objectives for Gameplay and Learning, In Karen Schrier (eds), Learning, Education and Games: Volume One: Curricular and Design Considerations, ETC Press, p. 227252. 